\title{
Nuclear Thermometers for Classical Novae
}

\section{Lori Downen ${ }^{* a, b}$ Christian Iliadis, ${ }^{a, b}$ Jordi José,,${ }^{c, d}$ and Sumner Starrfield}

${ }^{a}$ Department of Physics and Astronomy, University of North Carolina at Chapel Hill, Chapel Hill, NC 27599-3255, USA

${ }^{b}$ Triangle Universities Nuclear Laboratory, Durham, NC 27708-0308, USA

${ }^{c}$ Departament de Física i Enginyeria Nuclear, EUETIB, Universitat Politècnica de Catalunya, E-08036 Barcelona, Spain

'Institut d'Estudis Espacials de Catalunya, E-08034 Barcelona, Spain

${ }^{e}$ School of Earth and Space Exploration, Arizona State University, Tempe, AZ 85287-1404

E-mail: Indownen@physics.unc.edu, iliadis@unc.edu,

jordi.jose@upc.edu, starrfiedsophie.la.asu.edu

Classical novae are interesting phenomena that occur in binary systems containing a white dwarf and a main sequence (or Red Giant) companion. The resulting thermonuclear runaway can reach peak temperatures of several hundred million kelvin. Additionally, this explosion can display strong spectral lines of neon in the ejecta if the core of the white dwarf in this system consists of mainly neon and oxygen, leading these explosions to be termed "neon novae." For this work, the relationship between elemental abundances and the peak temperature during neon nova outbursts was explored, and this relationship was further investigated to determine any dependence on uncertain nuclear physics data. Four new hydrodynamic models of neon nova were developed, allowing for the determination of eight elemental abundance ratios that can be used as "nova thermometers": N/O, N/Al, O/S, S/Al, O/Na, Na/Al, O/P, and P/Al. Using a post-processing reaction network, it was determined that $\mathrm{N} / \mathrm{O}, \mathrm{N} / \mathrm{Al}, \mathrm{O} / \mathrm{Na}$, and $\mathrm{Na} / \mathrm{Al}$ had little dependence on uncertain rate information, introducing only $<30 \%$ variation in abundance ratio. The remaining thermometers, $\mathrm{O} / \mathrm{S}, \mathrm{S} / \mathrm{Al}, \mathrm{O} / \mathrm{P}$, and $\mathrm{P} / \mathrm{Al}$, showed a steeper dependence on peak temperature but were strongly dependent on the uncertain ${ }^{30} \mathrm{P}(\mathrm{p}, \gamma){ }^{31} \mathrm{~S}$ rate. Updated observed nova abundances were compiled and qualitatively compared to several nova thermometers. It was determined that several nova thermometers would benefit significantly from renewed efforts to reliably observe ejecta abundances and precisely quantify the ${ }^{30} \mathrm{P}(\mathrm{p}, \gamma){ }^{31} \mathrm{~S}$ reaction rate.

XII International Symposium on Nuclei in the Cosmos,

August 5-12, 2012

Cairns, Australia

\footnotetext{
*Speaker.
} 


\section{Introduction}

Classical novae are a type of stellar explosion that occur when a white dwarf and a companion star exist in a close binary system [1]. Leading up to this explosion, hydrogen-rich matter from the companion star accretes onto the surface of the white dwarf. This mixture of white dwarf and solar material undergoes hydrogen burning until a thermonuclear runaway takes place, ejecting matter into the interstellar medium. Spectroscopic analysis of this material reveals its composition in terms of elemental abundances [2,3, and references therein].

The study of classical novae is of significant interest for several reasons. Spectroscopic studies, after proper interpretation, constrain stellar evolutionary models by revealing the composition of the underlying white dwarf. Observed elemental abundances also provide a record of the thermonuclear runaway, including peak temperatures and expansion timescales, and have been used to constrain stellar explosion models [4]. Classical novae are noteworthy contributors to the chemical evolution of the Galaxy because they are major sources of ${ }^{13} \mathrm{C},{ }^{15} \mathrm{~N}$, and ${ }^{17} \mathrm{O}[5,6,7,2]$ as well.

An interesting subset of this phenomena, ONe novae, show strong spectral lines of neon in the ejected material [3] and are the focus of this work. These novae involve white dwarfs consisting primarily of oxygen and neon with a mass range of $\approx 1.1-1.4 \mathrm{M}_{\odot}$ [8, and references therein], making them significantly more massive than carbon-oxygen $(\mathrm{CO})$ composition white dwarfs, and reach peak temperatures of several hundred million kelvin.

These novae are not thoroughly understood however. Therefore, the goal of this work is to investigate if, and how, simulated elemental abundances in classical novae shells can effectively constrain crucial explosion parameters, most notably the peak temperature. More specifically, we seek to develop "nova thermometers": elemental abundance ratios with a strong correlation to peak temperature. The robustness of these thermometers is tested by varying relevant reaction rates within their uncertainties. Finally, the effectiveness of the thermometers is studied by qualitatively comparing their results to observational data.

\section{Method}

First, we generated a series of four new hydrodynamic models using the code SHIVA [6] with reaction rates and uncertainties adopted from STARLIB [9]. Our nuclear reaction network followed the evolution of 117 nuclides in the range from $\mathrm{H}$ to Ti through 635 nuclear processes, including weak interactions, reactions of type $(\mathrm{p}, \gamma),(\mathrm{p}, \alpha),(\alpha, \gamma)$, and their reverse reactions. The different models were generated for four white dwarf masses $\left(1.15 \mathrm{M}_{\odot}, 1.25 \mathrm{M}_{\odot}, 1.30 \mathrm{M}_{\odot}\right.$, and $\left.1.35 \mathrm{M}_{\odot}\right)$. We assumed a mixing fraction of $50 \%$ between accreted matter of solar composition and white dwarf matter prior to the outburst. The solar abundances are adopted from [10], while the white dwarf abundances are taken from the evolution of a $10 M_{\odot}$ star from the main sequence to the end of core carbon burning [11].

While hydrodynamic simulations were effective in exploring possible nova thermometers, investigating the sensitivity of any useful nova thermometer to nuclear reaction rate variations requires that a large number of simulations be performed in order to exhaust the nuclear physics parameter space. Full hydrodynamic model simulations are far too time-consuming for this purpose at present. Instead, we performed post-processing reaction network calculations, using time- 
temperature-density profiles adopted from the hydrodynamic models described above. Such calculations have been used successfully in previous reaction rate sensitivity studies of classical novae [12].

Previous investigations employed one-zone calculations, appropriate for the hottest zone close to the white dwarf surface only. However, it was important to perform post-processing calculations for all burning zones, matching the final abundances of the hydrodynamic models as closely as possible. We explored two extreme assumptions, the "no-mixing" and "instant-mixing" approximations, regarding the mixing of material between zones. In the first case, we performed postprocessing calculations for each burning zone separately and at the end of the calculation the elemental abundances were mass-averaged over all zones [13]. In the second case, the post-processing calculation is performed by replacing each local thermonuclear rate by its mass-weighted average over the convective region, implying that the turnover time is faster than the nuclear burning time [14]. The actual behavior probably lies somewhere between these prescriptions. Therefore, a third set of elemental abundances, the geometric mean of the abundances from the no-mixing and instant-mixing approximations, was derived.

More than 7,000 multi-zone network calculations for all hydrodynamic models were performed. For each reaction, the rates were multiplied by factors of 100, 10, 5, 2, 0.5, 0.2, 0.1, and 0.01 in succes- sive network calculations. Final elemental abundances were adopted from the mixing prescription that best approximated the results of a hydrodynamic model using recommended reaction rates. Final elemental abundances are then determine the impact of the actual reaction rate uncertainties, which were adopted from the STARLIB library.

\section{Results}

Using the SHIVA hydrodynamic simulations, we find that certain elements are overproduced $(\mathrm{N}, \mathrm{Si}, \mathrm{P}$, and $\mathrm{S})$, while others are depleted $(\mathrm{O}, \mathrm{Na}, \mathrm{Mg}$ ) versus their initial abundances. $\mathrm{N}, \mathrm{Na}, \mathrm{P}$, and $\mathrm{S}$ are particularly interesting as they monotonically increase with peak temperature, while $\mathrm{O}$ and $\mathrm{Al}$ are also of interest due to their monotonically decreasing behavior with peak temperature. Based on the hydrodynamic simulations, our first indication of useful thermometers, with a steep dependence on peak temperature, are the eight element ratios N/O, N/Al, O/S, S/Al, O/Na, Na/Al, $\mathrm{O} / \mathrm{P}$, and $\mathrm{P} / \mathrm{Al}$.

The elemental abundance ratios $\mathrm{O} / \mathrm{S}$ (decreasing) and $\mathrm{S} / \mathrm{Al}$ (increasing) display the strongest variation between peak temperatures of $228 \mathrm{MK}$ and $313 \mathrm{MK}$, amounting to about three orders of magnitude. Also, the abundance ratios $\mathrm{O} / \mathrm{P}$ (decreasing) and $\mathrm{P} / \mathrm{Al}$ (increasing) vary by more than two orders of magnitude. The other ratios $(\mathrm{O} / \mathrm{Na}, \mathrm{N} / \mathrm{Al}, \mathrm{N} / \mathrm{O}, \mathrm{Na} / \mathrm{Al})$ show variations by about one order of magnitude. The next step was to investigate how robust these element ratios are with regard to thermonuclear reaction rate variations.

The sensitivity of the nova thermometers to the nuclear physics input is shown in Figure 1. Elemental abundance (mass fraction) ratios are displayed versus peak temperature, for the nova models explored here. Interestingly, four of the thermometers (N/O, N/Al, O/Na, and Na/Al) reveal robust abundance ratios, with changes to abundance ratios less than $30 \%$. These ratios represent currently useful nova thermometers. However, only the first two (N/O and N/Al) involve elements that have so far been observed in neon nova shells. The last two involve sodium, which has been 


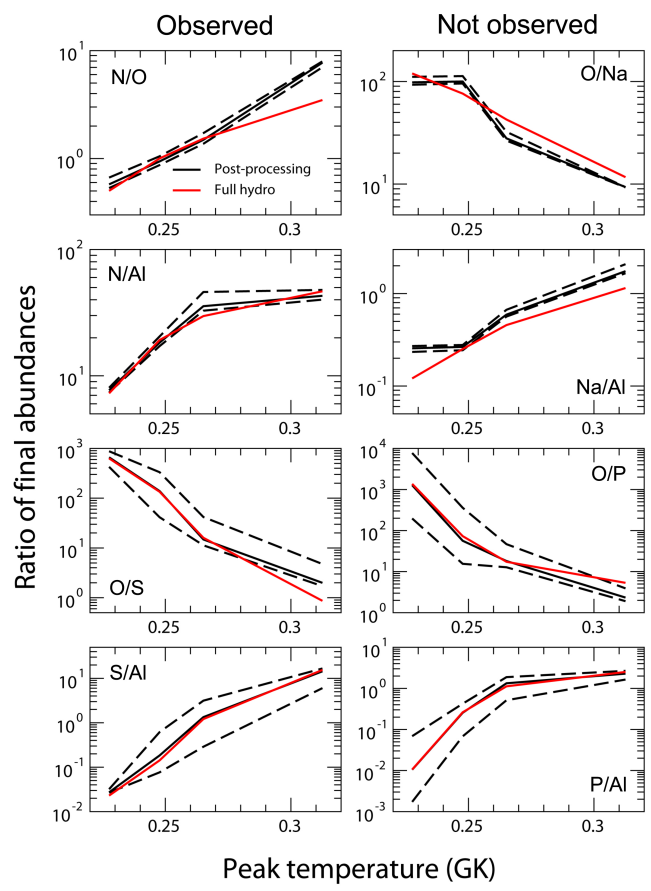

Figure 1: Eight ratios of elemental abundances (mass fractions), prime candidates for nova thermometers, that show a steep and monotonic dependence on peak temperature. Left Panel: All ratios shown in the left column involve elements that have been observed in neon nova shells. Right Panel: All ratios shown in the right column involve elements ( $\mathrm{Na}$ and $\mathrm{P}$ ) that have not been observed in neon nova shells.

searched for in infrared spectra but not yet detected. The use of the other four predicted elemental ratios $(\mathrm{O} / \mathrm{S}, \mathrm{S} / \mathrm{Al}, \mathrm{O} / \mathrm{P}$, and $\mathrm{P} / \mathrm{Al})$ as nova thermometers is somewhat limited. The first two of these ratios $(\mathrm{O} / \mathrm{S}$ and $\mathrm{S} / \mathrm{Al})$ involve elements observed in neon nova shells, while the other two ratios $(\mathrm{O} / \mathrm{P}$ and $\mathrm{P} / \mathrm{Al}$ ) involve phosphorous, which has not been successfully observed [15]. Thus, our results call for improved observations and spectral analysis of neon nova ejecta (i.e., for phosphorous). These ratios are also uncertain by factors of 3-6, primarily due to the uncertain ${ }^{30} \mathrm{P}(\mathrm{p}, \gamma){ }^{31} \mathrm{~S}$ reaction rate. Future laboratory measurements of nuclear reactions would clearly improve their utility as nova thermometers as well as the continued study of ${ }^{30} \mathrm{P}(\mathrm{p}, \gamma)^{31} \mathrm{~S}[16,17]$ in particular.

\section{Comparison of Our Predictions to Observations}

A first general impression can be obtained from the top panel of Figure 2, showing a threeelement abundance plot involving S, Al and O, i.e., S/Al vs. O/S. Our model predictions are shown as thick and thin solid, black lines (see Figure 1). The predicted O/S and S/Al abundance ratios have rather large uncertainties, which is reflected by the broad region between the two thin, solid lines. These uncertainties originate from the poorly known ${ }^{30} \mathrm{P}(\mathrm{p}, \gamma){ }^{31} \mathrm{~S}$ reaction rate (Figure 1). Only for a single neon nova, V838 Her (blue) [18], have all three elements been observed simultaneously. It is apparent in this case that the observational uncertainties are relatively small compared to the stellar model results. We conclude that the $\mathrm{O} / \mathrm{S}$ and $\mathrm{S} / \mathrm{Al}$ abundance ratios will become more useful thermometers for neon novae when the ${ }^{30} \mathrm{P}(\mathrm{p}, \gamma){ }^{31} \mathrm{~S}$ reaction rate can be determined experimentally 
and $\mathrm{Al}$ and $\mathrm{S}$ are simultaneously observed in additional novae.

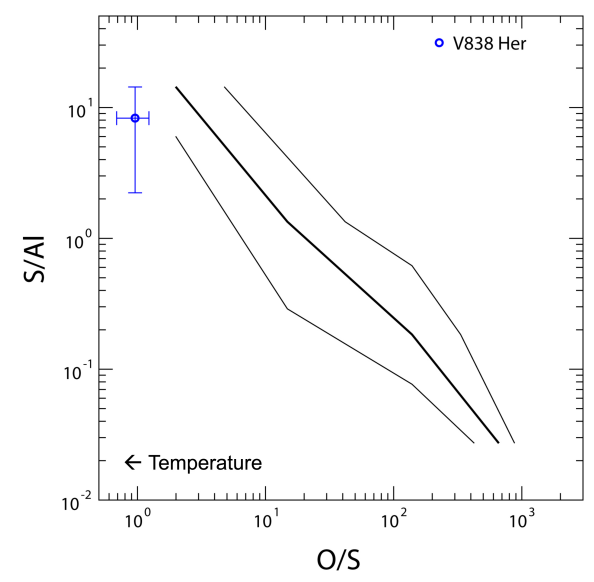

Figure 2: Three-element plot of $\mathrm{O}, \mathrm{Al}$, and $\mathrm{S}$ used to compare elemental ratios predicted by post-processing calculations to that of classical nova observations. Note that peak temperature in the simulation results increases from right to left in this figure.

\section{Conclusions}

One goal of the present work was to investigate how useful elemental abundances are for determining the peak temperature, which is strongly correlated with the underlying white dwarf mass, achieved during neon nova outbursts. Another aim was to determine how robust such "nova thermometers" are with respect to currently uncertain nuclear physics input. We derived updated observed abundances for several neon novae and performed new hydrodynamic simulations of neon novae, with peak temperatures in the range of $228 \mathrm{MK}$ to $313 \mathrm{MK}$. We found that the most useful thermometers are N/O, N/Al, O/S, S/Al, O/Na, Na/Al, O/P, and P/Al.

Next, we investigated the sensitivity of these nova thermometers to thermonuclear reaction rate variations. The ratios $\mathrm{N} / \mathrm{O}, \mathrm{N} / \mathrm{Al}, \mathrm{O} / \mathrm{Na}$, and $\mathrm{Na} / \mathrm{Al}$ are robust, meaning they are affected by uncertain reaction rates by less than $30 \%$. The last two ratios, $\mathrm{O} / \mathrm{Na}$ and $\mathrm{Na} / \mathrm{Al}$, are not currently useful as sodium has not yet been detected in classical nova spectra although it has been searched for. We also find that for these four elemental abundance ratios the dependence on peak temperature is not as strong as for some other ratios. The thermometers $\mathrm{O} / \mathrm{S}, \mathrm{S} / \mathrm{Al}, \mathrm{O} / \mathrm{P}$, and $\mathrm{P} / \mathrm{Al}$ reveal far steeper monotonic dependences on peak temperature. However, their current drawback is the strong dependence on the uncertain ${ }^{30} \mathrm{P}(\mathrm{p}, \gamma){ }^{31} \mathrm{~S}$ reaction rate. Thus, our study provides additional motivation for new laboratory measurements of this crucial nuclear reaction. Also, the last two of these ratios, $\mathrm{O} / \mathrm{P}$ and $\mathrm{P} / \mathrm{Al}$, involve phosphorus, another element which has not been detected in classical nova spectra.

\section{Acknowledgments}

This work was supported in part by the US Department of Energy (DE-FG02-97ER41041), the National Science Foundation (AST-1008355), the Spanish MICINN grants (AYA2010-15685, 
EUI2009-04167), the E.U. FEDER funds, the ESF EUROCORES Program EuroGENESIS, and by Arizona State University funding from the National Science Foundation and the National Aeronautics and Space Administration.

\section{References}

[1] M. Bode, A. Evans, Classical Novae (2nd ed.), Cambridge University Press, Cambridge 2008.

[2] S. Starrfield, C. Iliadis, W. R. Hix, Thermonuclear processes, in Classical Novae (2nd ed.), Cambridge University Press, Cambridge 2008.

[3] R. D. Gehrz, J. W. Truran, R. E. Williams, S. Starrfield, Nucleosynthesis in Classical Novae and Its Contribution to the Interstellar Medium, PASP 110 (3) 1998.

[4] S. Starrfield, W. M. Sparks, J. W. Truran, M. C. Wiescher, The effects of new nuclear reaction rates and opacities on hydrodynamic simulations of the nova outburst, ApJS 127 (485) 2000.

[5] A. Kovetz, D. Prialnik, The composition of nova ejecta from multicycle evolution models, ApJ 477 (356) 1997.

[6] J. José, M. Hernanz, Nucleosynthesis in classical novae: CO versus ONe white dwarfs, ApJ 494 (680) 1998.

[7] J. José, M. Hernanz, C. Iliadis, Nucleosynthesis in classical novae, Nucl. Phys. A 777 (550) 2006.

[8] L. G. Althaus, A. H. Córsico, J. Isern, E. Garcia-Berro, Evolutionary and pulsational properties of white dwarf stars, A\&A 18 (471) 2010.

[9] C. Iliadis, A. Champagne, A. Chieffi, M. Limongi, The effects of thermonuclear reaction rate variations on ${ }^{26}$ Al production in massive stars: a sensitivity study, ApJS 193 (16) 2011.

[10] K. Lodders, H. Palme, H.-P. Gail, Abundances of the elements in the solar system in Landolt-Börnstein New Series VI/4B, Springer, Berlin 2009.

[11] C. Ritossa, E. García-Berro, I. Iben, Jr.,On the Evolution of Stars That Form Electron-degenerate Cores Processed by Carbon Burning. II. Isotope Abundances and Thermal Pulses in a $10 M_{\odot}$ Model with an ONe Core and Applications to Long-Period Variables, Classical Novae, and Accretion-Induced Collapse, ApJ 460 (489) 1996.

[12] C. Iliadis, A. Champagne, J. José, S. Starrfield, P. Tupper, The Effects of Thermonuclear Reaction-Rate Variations on Nova Nucleosynthesis: A Sensitivity Study, ApJS 142 (105) 2002.

[13] M. S. Smith, W. R. His, S. Parete-Koon, L. Dessieux, M. W. Guidry, D. W. Bardayan, S. Starrfield, D. L. Smith, A. Mezzacappa, Nova Nucleosynthesis Calculations: Robust Uncertainties, Sensitivities, and Radioactive Ion Beam Measurements, Proceedings of the International Conference on Classical Nova Explosions 637 (161) 2002.

[14] D. Prialnik, The evolution of a classical nova model through a complete cycle, ApJ 310 (222) 1986.

[15] S. Shore, private communication 2012.

[16] D. Irvine, ${ }^{30} P(p, \gamma)^{31} S$ Reaction Rate for Classical Novae: An Indirect Approach, Journal of Physics: Conference Series 381 (012144) 2012.

[17] A. Parikh, K. Wimmer, T. Faestermann, et al., Improving the ${ }^{30} P(p, \gamma)^{31} S$ rate in oxygen-neon novae: Constraints on $J^{\pi}$ values for proton-threshold states in ${ }^{31} S$, Phys. Rev. C 83 (045806) 2011.

[18] G. J. Schwarz, S. N. Shore, S. Starrfield, K. M. Vanlandingham, Abundance Analysis of the Extremely Fast ONeMg Novae V838 Herculis and V4160 Sagittarii, ApJ 657 (453) 2007. 